\title{
Respiratory rehabilitation for patients with COVID-19 infection and chronic respiratory failure: a real-life retrospective study by a Lombard network
}

\author{
Michele Vitacca $^{1}$, Beatrice Salvi ${ }^{1}$, Marta Lazzeri ${ }^{2}$, Elisabetta Zampogna ${ }^{3}$, Giancarlo Piaggi ${ }^{4}$, \\ Piero Ceriana $^{5}$, Serena Cirio ${ }^{5}$, Luigino Rizzello ${ }^{6}$, Grazia Lacala ${ }^{6}$, Angelo Longoni ${ }^{7}$, Vittoria Galimberti ${ }^{8}$, \\ Patrizia D'Ambrosio $^{8}$, Enrica Pavesi ${ }^{9}$, Giuseppe La Piana ${ }^{9}$, Antonella Sanniti ${ }^{10}$, Alessandro Morandi ${ }^{10}$, \\ Manoel Vallet $^{11}$, Mara Paneroni ${ }^{1}$ on behalf of AIPO, SIP and ARIR societies
}

\begin{abstract}
${ }^{1}$ Respiratory Rehabilitation, ICS Maugeri IRCCS, Institute of Lumezzane (BS); ${ }^{2}$ Department of Cardiothoracic and Vascular Surgery, ASST Grande Ospedale Metropolitano Niguarda, Milan; ${ }^{3}$ Respiratory Rehabilitation, ICS Maugeri IRCCS, Institute of Tradate (VA); ${ }^{4}$ Respiratory Rehabilitation, ICS Maugeri IRCCS, Institute of Montescano (PV); ${ }^{5}$ Respiratory Rehabilitation, ICS Maugeri IRCCS, Institute of Pavia; ${ }^{6}$ Respiratory Rehabilitation, ICS Maugeri IRCCS, Institute of Milan; ${ }^{7}$ ASST Lariana - P.O. Sant'Antonio Abate, Cantù (MO); ${ }^{8}$ Hospital San Giuseppe, Milan; ${ }^{9}$ Hospital Santa Marta, Rivolta d'Adda (CR); ${ }^{10}$ Casa di Cura Ancelle della Carità, Fondazione Teresa Camplani, Cremona; ${ }^{11}$ Casa di Cura Ancelle della Carità, Fondazione Teresa Camplani, Brescia, Italy
\end{abstract}

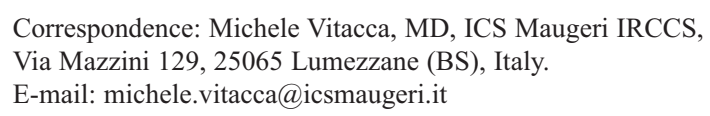

Conflict of interest: The authors declare that they have no competing interests, and all authors confirm accuracy.

Availability of data and materials: The data used to support the findings of this study are available from the corresponding author on reasonable request.

Ethics approval and consent to participate: The study was approved by the Istituti Clinici Scientifici (ICS) Maugeri Ethics Committee (CEC 2279; March 12 $\left.{ }^{\text {th }}, 2020\right)$. At admission to institutions, patients gave informed consent for the scientific use of their data. As retrospective analysis, the study was not registered.

Acknowledgments: We thank Manoel Vallet MD for English revision, Laura Comini and Adriana Olivares for editing revision.

Received for publication: 14 June 2021.

Accepted for publication: 20 December 2021.

Publisher's note: All claims expressed in this article are solely those of the authors and do not necessarily represent those of their affiliated organizations, or those of the publisher, the editors and the reviewers. Any product that may be evaluated in this article or claim that may be made by its manufacturer is not guaranteed or endorsed by the publisher.

${ }^{\circ}$ Copyright: the Author(s), 2021

Licensee PAGEPress, Italy

Monaldi Archives for Chest Disease 2022; 92:1975

doi: 10.4081/monaldi.2021.1975

This article is distributed under the terms of the Creative Commons Attribution-NonCommercial International License (CC BY-NC 4.0) which permits any noncommercial use, distribution, and reproduction in any medium, provided the original author(s) and source are credited.

\begin{abstract}
The Lombardy region has been one of the areas most affected by the COVID-19 pandemic since the first months of 2020, providing real-life experiences in the acute phase. It is unclear how the respiratory rehabilitation network responded to this emergency. The aims of this retrospective study were: i) to analyze clinical, functional, and disability data at admission; ii) describe assessment tools and rehabilitative programs; iii) evaluate improvement after rehabilitation. The study was conducted on data collected from ten pulmonary rehabilitation centers in Lombardy, between the period of March $1^{\text {st }} 2020$ to March $1^{\text {st }}$ 2021 , in patients with respiratory failure recovering from COVID19 both at admission and discharge. The study included demographics, comorbidities, nutritional status, risk of falls, disability status (Barthel index; Short Physical Performance Battery (SPPB); 6 minutes walking test (6MWT), symptoms (dyspnoea with Barthel Dyspnoea and MRC Dyspnoea Scale), length of stay, discharge destination, need for mechanical ventilation, respiratory function, assessment/outcomes indices, and prescribed rehabilitative programs. 413 patients were analyzed. Length of stay in acute and rehabilitative units was less than 30 days. Fifty $\%$ of patients used non-invasive ventilation during their stay. Functional status was mildly compromised for forced volumes and oxygenation, while severely compromised for diffusion capacity. Independency was low while physical performance status very low. At discharge, $318(77 \%)$ patients were sent home, 83 (20.1\%) were transferred to an acute unit and $12(2.9 \%)$ passed away. Barthel Index and 6MWT were the most used tests, while MRC score was the least used outcome parameter. The 5 main rehabilitative activities were walking $(90.8 \%)$, transfer from bed to armchair (77.5\%), limb mobilization in bed $(76 \%)$, balance $(71.2 \%)$, and cycle-ergometer or treadmill (43.1\%). A huge difference was found in admission, discharge, and delta change among different rehabilitative centers. When available, all outcomes showed a significant improvement. With the limitation of a retrospective study with a clear amount of missing data, COVID-19 subjects admitted to rehabilitative centers presented a reduced physical performance, symptoms of dyspnoea, and severe disability. The 6MWT and Barthel index were the most used measurement.
\end{abstract}




\section{Introduction}

The pulmonary disease developed by subjects infected with COVID-19 virus proved to be extremely heterogeneous in terms of clinical presentation, disability, outcomes and prognosis [1-3]. The possible outcomes after hospitalization are residual symptomatic limitations, functional damage, the appearance of disability, reduced exercise tolerance and neuropsychological damage [1-3]. Panels of experts have proposed rehabilitation paths [4-6] and some scientific reports have already highlighted the benefits of post-COVID-19 pneumonia rehabilitation programs [7-9]. In Lombardy (the most populated Italian region with more than 10 million residents) there are plenty of pulmonary rehabilitation opportunities, but they vary in volume of activities after acute hospitalisation. During the first months of 2020, Lombardy has been one of the areas most affected by the COVID-19 pandemic, providing real life experiences both in the post-acute phase [10] and in rehabilitation [7, 8]. It is unclear how the respiratory rehabilitation network responded to this emergency. To help clarify this, three scientific respiratory societies - ARIR (Associazione Riabilitatori dell'Insufficienza Respiratoria), AIPO (Associazione Italiana Pneumologi Ospedalieri) and SIP (Società Italiana di Pneumologia) - have proposed a "real-life" evaluation that investigates activities dedicated to post-COVID-19 patients.

The aims of this retrospective study were to: i) analyse clinical, functional and disability data at admission; ii) describe the measurement tools and the proposed rehabilitative program; iii) evaluate improvement after rehabilitation for patients with respiratory failure as a consequence of SARS-CoV-2 pneumonia.

\section{Ethical statement}

The study was approved by the Istituti Clinici Scientifici (ICS) Maugeri Ethics Committee (CEC 2279; March 12 $\left.{ }^{\text {th }}, 2020\right)$. At admission to institutions, patients gave informed consent for the scientific use of their data. As retrospective analysis, the study was not registered.

\section{Patients}

This study was conducted on the Automated Integrated Health Care Records databases on available data collected from ten pulmonary rehabilitation centres in Lombardy: ICS Maugeri Hospitals (Lumezzane, Tradate, Pavia, Montescano, Milan); Sant'Antonio Abate Hospital, Cantù; San Giuseppe Hospital, Milan; Santa Marta Hospital, Rivolta d'Adda; Casa di Cura Ancelle della Carità, Cremona and Brescia; Fondazione Teresa Camplani (FTC), Cremona and Brescia (Figure 1).

The study period was from March $1^{\text {st }} 2020$ to March $1^{\text {st }} 2021$. Included patients were recovering from pneumonia related to COVID-19 and consecutively admitted for inpatient pulmonary rehabilitation. Concerning provenience, these patients came from intensive and sub-intensive care units, general wards or home. They required oxygen supply, non-invasive ventilation (NIV) or invasive mechanical ventilation (IMV) $[11,12]$. There were no specific exclusion criteria.

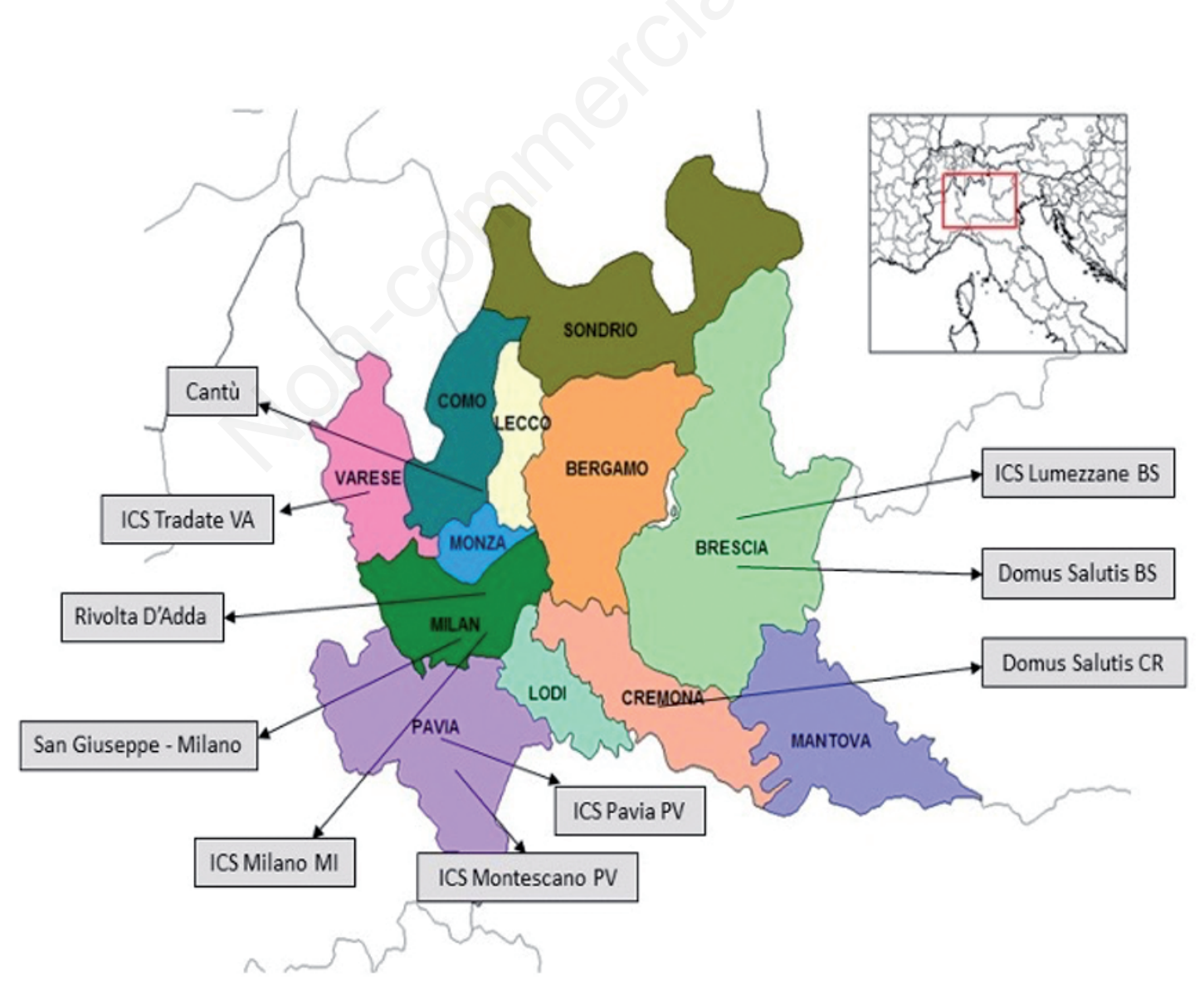

Figure 1. Distribution of Lombardy rehabilitative network. 


\section{Measurements}

Data was recorded when patients were admitted to the rehabilitative hospital and on discharge. At admission the following data were collected: demographics, length of stay in acute hospital, use of oxygen supply, use of NIV or IMV and diagnosis of comorbidities by the Cumulative Illness Rating Scale (CIRS) [13].

Information about ADL independency and disability was recorded as follows: motor performance was assessed by the Barthel index (BI) [14], physical performance was assessed by the Short Physical Performance Battery (SPPB) [15,16], exercise tolerance was assessed by the 6 minutes walking test (6MWT) [17], dyspnoea during ADLs was assessed by the Barthel dyspnoea [18] and Medical Research Council (MRC) dyspnoea scale [19].

Data related to nutritional status (Malnutrition Universal Screening Tool - MUST) and risk of falls (Morse fall scale) were also collected at admission.

\section{Pulmonary rehabilitation}

In accordance with the Italian Position Paper, a multidisciplinary program was applied in all centres [20]. The best rehabilitation program for the patient was selected according to age, clinical severity, length of immobilization and comorbidities. Different modality of intervention, type, intensity and timing were considered. All patients began with a 20-min session per day and continued with a range of time between 30-60 min/session for once or twice per day. Sessions could be individual or in groups. Individual sessions involved pas- sive mobilization, bed position changes, stand-up exercises, active exercises, free walking or walking with assistance, peripheral limb muscle strengthening and balance training. Group sessions involved strengthening, aerobic and balance exercises. Patients with a higher physical autonomy were also trained with cycle-ergometer or treadmill. Each patient started with a zero-training workload and progressively increased it until patient scored four or five points at dyspnoea or leg fatigue using the Borg scale [21]. During rehabilitation, patients were evaluated daily in order to tailor intervention type, intensity and/or length of the program.

\section{Statistical analysis}

Data were registered on a dedicated database $\left(\right.$ Excel $^{\circledR}$, Microsoft, Redmond, WA, USA). Continuous variables were expressed as mean and standard deviation (SD) and binary or categorical outcomes were described as percentage (\%). Paired $t$-test was used for pre- to postcomparison; statistically significant value was set at $\mathrm{p}<0.05$.

\section{Results}

This study included 413 patients coming from the ten rehabilitation centres listed above. Demographics, physiological, and clinical characteristics of patients are shown in Table 1.

Most of the patients arrived in the rehabilitation units from acute hospital, while only $7.7 \%$ of patients arrived from home. Length of stay in an acute unit was less than 30 days for all

Table 1. Baseline demographics, clinical and functional data.

\begin{tabular}{|c|c|}
\hline Patients, $n$ & 413 \\
\hline Sex, n (\%) & 286 (69.2\%) M; 127 (30.8\%) F \\
\hline Age, years & $70.97 \pm 11.76$ \\
\hline BMI, $\mathrm{kg} / \mathrm{m}^{2}$ & $26.40 \pm 6.0$ \\
\hline CIRS, score (279 pts) & $3.03 \pm 1.90$ \\
\hline Place of acute phase management, $n(\%)$ & hospital: 381 (92.3\%); home: 32 (7.7\%) \\
\hline Length of stay in acute unit, day & $24.77 \pm 19.04$ \\
\hline Tracheostomy, n (\%) & No=367 (88.9\%); Yes=46 (11.1\%) \\
\hline CPAP/NIV, n (\%) & No=286 (69.2\%); Yes=127 (30.8\%) \\
\hline Length of stay in rehabilitative unit, day & $25.73 \pm 17.46$ \\
\hline $\mathrm{FEV}_{1}, \%$ prd (50 pts) & $75.88 \pm 17.89$ \\
\hline FVC, \% prd (50 pts) & $73.66 \pm 19.83$ \\
\hline IC, $\%$ prd (50 pts) & $63.14 \pm 22.89$ \\
\hline DLCO, \% prd (26 pts) & $50.35 \pm 22.45$ \\
\hline $\mathrm{PaO}_{2} / \mathrm{FiO}_{2}$ at admission (181 pts) & $307.81 \pm 88.21$ \\
\hline Barthel index, score (162 pts) & $56.81 \pm 31.49$ \\
\hline SPPB, score (143 pts) & $4.61 \pm 4.04$ \\
\hline Barthel dyspnoea, score (127 pts) & $27.82 \pm 22.46$ \\
\hline 6MWT, meters (199 pts) & $215.77 \pm 146.95$ \\
\hline MRC, score (145 pts) & $2.66 \pm 1.07$ \\
\hline Place of discharge, n (\%) & dead: 12 (2.9\%); acute unit: 83 (20.1\%); home: 318 (77.0\%) \\
\hline
\end{tabular}


patients. All patients suffered from respiratory failure and many of them needed NIV; $11.14 \%$ of patients were admitted with tracheostomy and needing IMV. At least for the patients in which functional data were available, respiratory functional status seemed mildly compromised for lung function parameters and oxygenation, while severely impaired for diffusion capacity. At admission, at least for the patients in which disability data were available, patients presented high disability and a very low physical performance status assessed by Barthel index and SPPB, respectively. Barthel dyspnoea and MRC showed important dyspnoea during ADLs. Again, at least for the patients who performed the 6MWT, severe reduction in effort tolerance was found at the beginning of the rehabilitation program. On discharge the majority of patients were sent home and only a small group were transferred to an acute unit or passed away. Table 2 shows assessment/evaluation tests done by each centre according to habits, standards and organisational availability. BI and 6MWT were the most used, while MRC score was the less used outcome parameter.

Figure 2 shows percentage of patients admitted to the 5 main rehabilitative programs (passive/active limb mobilisation in bed; transfer from bed to armchair; walking; balance; cycle-ergometer or treadmill). On discharge $91 \%$ of patients were able to walk with or without assistance. Patients performed a dedicated program to walking in $90.8 \%$, transferring from bed to armchair in $77.5 \%$, limb bed mobilisation (76\%), balance in $71.2 \%$ and cycle-ergometer or treadmill training in $43.1 \%$.

Table 3 shows a huge difference in admission, discharge and subsequent delta improvement in 6MWT, SPPB and Barthel index among different rehabilitative centres. In the whole sample, for motor Barthel index a delta improvement of $21.96 \pm 24.74$ score was obtained. The hospitals with the higher improvements were ICS Lumezzane and FTC Brescia. For SPPB, a delta of 3.37 score was obtained. The hospital with the highest performance was ICS of Tradate. For 6MWT a delta of 105.57 meters was calculated. FTC Brescia was the hospital with the best performance.

Table 4 shows differences in admission, discharge and subsequent delta improvement in MRC and Barthel dyspnoea among centres. For MRC score a delta of 0.96 was obtained. The ICS of Lumezzane was the hospital with the best result. For Barthel dyspnoea, a delta of 14.43 was reached; the hospital with the best

Table 2. Assessment/outcomes indices used by different network hospitals.

\begin{tabular}{|c|c|c|c|c|c|c|c|}
\hline Rehabilitation Unit & Barthel index & MORSE & MUST & SPPB & MRC & Barthel dyspnoea & $6 \mathrm{MWT}$ \\
\hline ICS Milano & $X$ & $\mathrm{X}$ & $X$ & & & $x$ & \\
\hline ICS Lumezzane & $X$ & $X$ & $X$ & $X$ & $X$ & $X$ & $X$ \\
\hline ICS Montescano & $\mathrm{X}$ & $\mathrm{X}$ & 8 & $X$ & & $X$ & \\
\hline ICS Pavia & $X$ & $X$ & $X$ & $X$ & & $X$ & $X$ \\
\hline ICS Tradate & $\mathrm{X}$ & $X$ & $\mathrm{X}$ & $X$ & & & $X$ \\
\hline Sant'Antonio Abate, Cantù & & & & & & & $X$ \\
\hline FTC, Cremona & $X$ & & & & & $X$ & $X$ \\
\hline San Giuseppe, Milan & $X$ & & & & $X$ & & $X$ \\
\hline FTC, Brescia & $\mathrm{X}$ & (a) & & & & & $\mathrm{X}$ \\
\hline Santa Marta, Cremona & & $X$ & & $\mathrm{X}$ & $X$ & & $X$ \\
\hline
\end{tabular}

MORSE, Morse fall scale; MUST, malnutrition universal screening tool; SPPB, short physical performance battery; MRC, MRC dyspnoea scale; 6MWT, six-minute walking test; FTC, Fondazione Teresa Camplani.

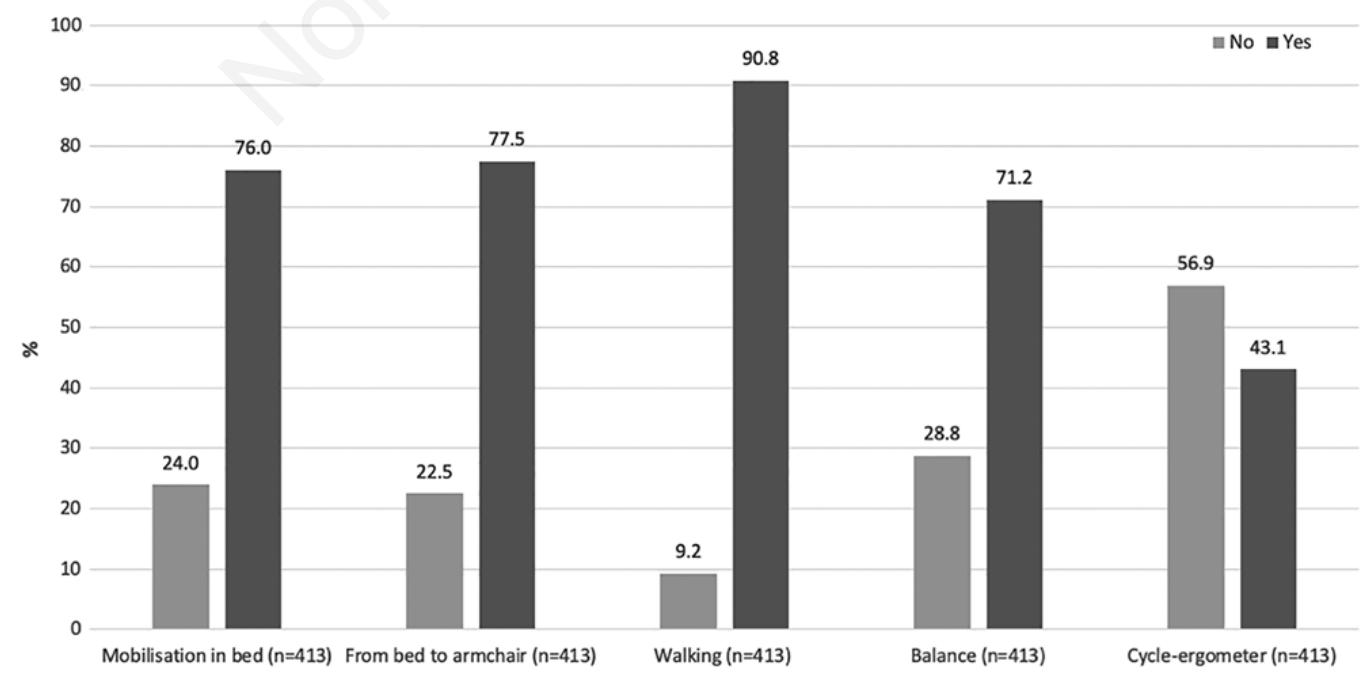

Rehabilitative program

Figure 2. Percentage of patients admitted to the 5 main rehabilitative programs. 


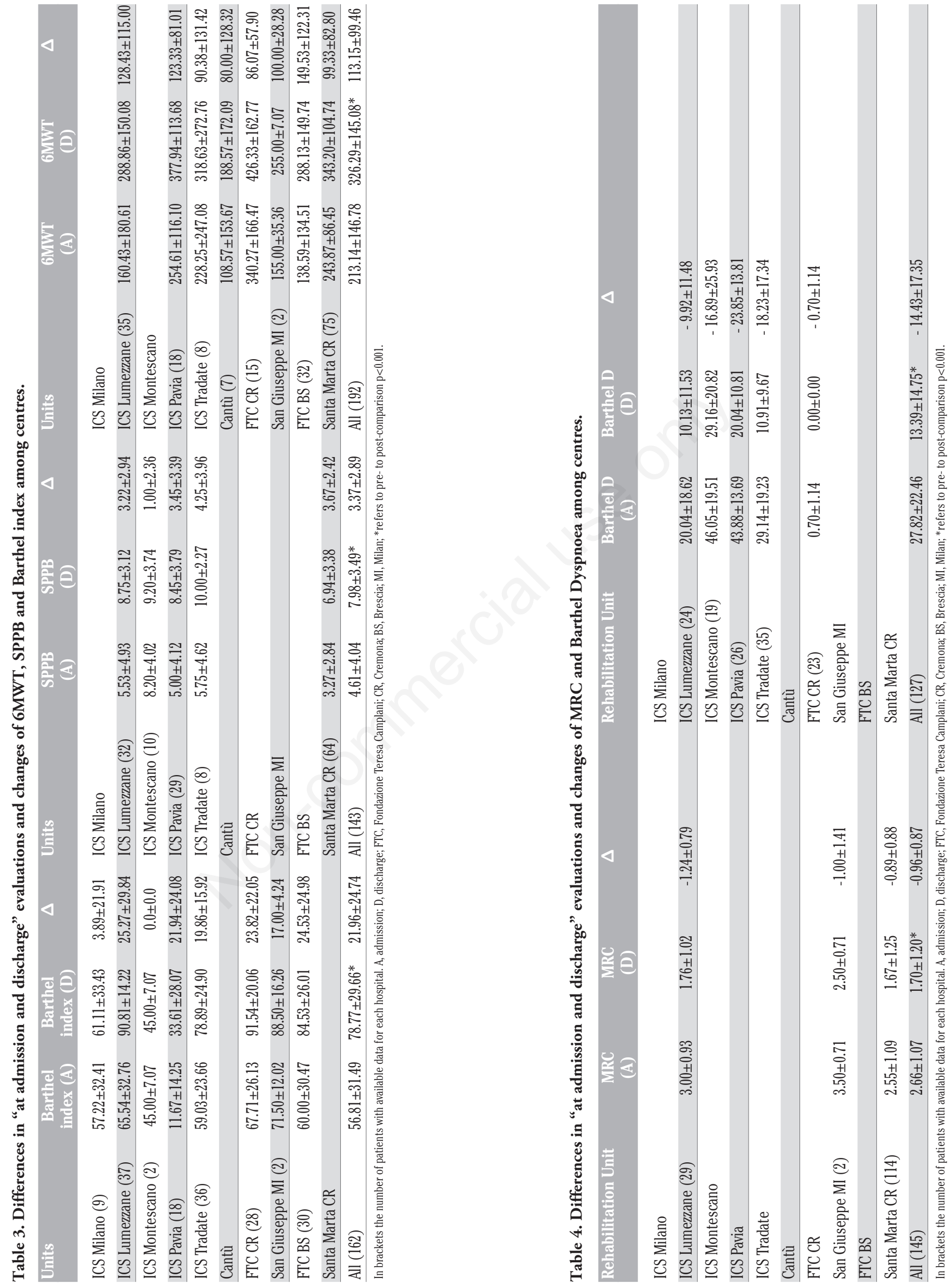


result was the Institute of Pavia. Oxygenation $\left(\mathrm{PaO}_{2} / \mathrm{FiO}_{2}\right)$ improved in all 181 patients (from $307.81 \pm 88.21$ to $330.97 \pm 76.83$; $\mathrm{p}<0.001)$.

\section{Discussion}

This study has shown, in a large sample of COVID-19 subjects that: i) patients with respiratory failure as a consequence of SARSCoV-2 pneumonia and admitted in rehabilitative centres showed a reduced physical performance, key symptoms and severe disability at admission; ii) the most used measurement tools were 6MWT and Barthel Index, while the majority of proposed programs used walking assistance; iii) the majority of patients improved all rehabilitative outcomes.

Pulmonary rehabilitation is possible and effective in patients recovering from COVID-19: our findings may be useful to guide clinicians, politicians and health organisations to follow up and support management of patients surviving COVID-19.

\section{Baseline assessment}

It is necessary to have tools that objectively measure the functional consequences of COVID-19 disease in the short- and longterm. It has been reported that post-COVID-19 patients can have an impaired physical function when they are discharged, even after early mobilization [22]. Our results confirm these observations and extend to more severe patients directly transferred from acute care hospitals. By comparison [22], our patients suffered from more severe acute conditions shown by longer length of stay in acute care hospitals and by the high proportion of patients receiving mechanical ventilation as Invasive (including some tracheostomized) or NIV. The level and severity of comorbidities, as assessed by the CIRS index, was similar to those of patients reported. Thus, the need for validated outcome measures is of utmost importance. Standardisation of measures would allow to make better comparisons among studies and different follow-up timepoints. The Barthel index and the six minutes walking test were the most used during patient stay in our network. The Barthel index was mainly used in the acute phase, whereas the 6MWT was used in interventional and follow-up studies. The 6MWT is the gold standard field exercise test and it has been validated for most chronic lung diseases. Eight centres out of ten used 6MWT to evaluate patients before and after rehabilitation. This test is sensitive, reproducible, easy to perform, and does not require any specialized equipment. In routine clinical practice, the Barthel Index is the most widely used scale to measure patients' motor and functional disabilities in activities of daily living (ADL). This index was developed for chronic and long-term hospital patients with neurological diseases to examine their performance before and after treatment. Five hospitals used SPPB test: this is a standardized objective tool which is rapid and simple to conduct and less influenced by cultural and educational background than other selfreport measures. It has also been shown that SPPB is significantly related to the capacity to perform ADLs, such as changing and maintaining body position, carrying, moving, and handling objects, or walking and gait pattern. Several instruments are commonly used to measure different domains of dyspnoea such as sensory-perceptual experience, affective distress, symptom impact or burden. The most used scale to assess dyspnoea in daily life was the modified Medical Research Council (mMRC) scale. Curci et al. found the most severe score (level 5) in 87.5 and $90.2 \%$ of COVID-19 patients [23]. Huang et al. [1] reported that, 6 months following disease onset, $26 \%$ of patients had mMRC levels greater than 1. Only one study [25] used the Barthel dyspnoea index, in a rehabilitative setting, and reported moderate levels of dyspnoea during activities of daily life.

\section{Rehabilitative effects}

Our pulmonary rehabilitation program was inspired by the Italian Position Paper dedicated to COVID-19 rehabilitation [5]. In real life, type, intensity, timing, and modality of intervention were tailored to the individual patients and according to local organization's protocol. The Sant'Antonio Abate Hospital (Cantù), the San Giuseppe Hospital (Milan), the FTC (Cremona and Brescia) and the ICS Maugeri in Lumezzane admitted the most complicated patients with a very low level of effort tolerance. An improvement in 6MWT of 105.57 meters in the whole group confirmed that the majority of patients reached the minimum clinically significant difference of 54 meters proposed for COPD patients. According to Perera et al. [26] one point is considered as the minimum clinically important difference (MCID) for SPPB and all studied patients who underwent this test got better after the rehabilitation program. Another tool is the MRC questionnaire, used mainly in COPD patients to measure perceived respiratory disease in activity of daily living. The MRC questionnaire was also only used at ICS Maugeri Lumezzane and San Giuseppe Milan resulting in an improvement over the rehabilitation program. Barthel dyspnoea is another questionnaire to assess patient's respiratory disease in activity of daily living. Four centres used it with very different scores at admission. ICS Maugeri Montescano and ICS Maugeri Pavia had the highest score showing a very high level of dyspnoea, while patients at FTC Cremona presented low level of dyspnoea. As a consequence, the first two centres had a better improvement over rehabilitation program. A wide range of different impairment in physical performance and in amount of response to rehabilitation programs was reported with the use of these tools. Differences among centres may be explained by different volumes of patients admitted, different admission severity levels and different assessment tools compared with different organisations offering rehabilitative programs.

\section{Practical implications}

The presented data shows the strong response, efficiency and effectiveness of the Lombard respiratory rehabilitation network, although still marked by a strong organizational heterogeneity and entry criteria. These pathways are often underutilized due to poor economic recognition though the enormous consumption of resources that specialist rehabilitation requires for severely chronically unstable and disabled patients [27]. In the era of the COVID-19 pandemic, the Lombard rehabilitation network offered strong support for severely affected COVID-19 patients to improve disability, quality of life and symptoms. The findings show the need to increase and develop the model of clinical care networks and emphasize the integration between hospital and territorial services in order to combine continuity of care and sustainability of the system. 


\section{Limitations of the study}

This design allowed including a large number of patients but, due to the retrospective nature of the study, a lot of data was missing and this is an important limitation. The results of an uncontrolled study may be difficult to interpret: a positive effect in the long-term follow-up of these patients without a rehabilitative intervention may not be excluded. An untreated group would be unethical given the undisputed benefits of pulmonary rehabilitation or simple physical activity.

\section{Conclusions}

This study has shown, in a large sample of COVID-19 subjects that, patients admitted in rehabilitative centres present a reduced physical performance, key symptoms and severe disability. 6MWT and Barthel index as measurement tools and walking as a program are the most used during the rehabilitative phase. The majority of patients improved in all outcomes with high variability among participating centres. Better standardisation is mandatory for future studies with higher methodological quality with the choice, timing and interpretation of measures of physical performance and rehabilitative prescription.

\section{References}

1. Huang C, Huang L, Wang Y, et al. 6-month consequences of COVID-19 in patients discharged from hospital: a cohort study. Lancet 2021;397:220-32.

2. Wang Z, Deng H, Ou C, Liang J, et al. Clinical symptoms, comorbidities and complications in severe and non-severe patients with COVID-19: A systematic review and meta-analysis without cases duplication. Medicine (Baltimore) 2020;99 e23327.

3. Paneroni M, Simonelli C, Saleri M, et al. Muscle strength and physical performance in patients without previous disabilities recovering from COVID-19 pneumonia. Am J Phys Med Rehabil 2021;100:105-9.

4. Lazzeri M, Lanza A, Bellini R, et al. Respiratory physiotherapy in patients with COVID-19 infection in acute setting: a Position Paper of the Italian Association of Respiratory Physiotherapists (ARIR). Monaldi Arch Chest Dis 2020;90:1285.

5. Vitacca M, Carone M, Clini EM, et al. Joint statement on the role of respiratory rehabilitation in the COVID-19 crisis: the Italian position paper. Respiration 2020;99:493-9.

6. Spruit MA, Holland AE, Singh SJ, et al. COVID-19: interim guidance on rehabilitation in the hospital and post-hospital phase from a European Respiratory Society and American Thoracic Society coordinated international task force. Eur Respir J 2020;56:2002197.

7. Zampogna E, Paneroni M, Belli S, et al. Pulmonary rehabilitation in patients recovering from COVID-19. Respiration 2021;100:416-22.

8. Ceriana P, Vitacca M, Paneroni M, et al. Usefulness of step down units to manage survivors of critical Covid-19 patients. Eur J Intern Med 2021;88:126-8.

9. Liu K, Zhang W, Yang Y, et al. Respiratory rehabilitation in elderly patients with COVID-19: A randomized controlled study. Complement Ther Clin Pract 2020;39:101166.

10. Vitacca M, Migliori GB, Spanevello A, et al. Management and outcomes of post-acute COVID-19 patients in Northern Italy. Eur J Intern Med 2020;78:159-60.

11. Ceriana P, Nava S, Vitacca M, et al. Noninvasive ventilation during weaning from prolonged mechanical ventilation. Pulmonology 2019;25:328-33.

12. Maestri R, Bruschi C, Fracchia C, et al. Physiological and clinical characteristics of patients with COPD admitted to an inpatient pulmonary rehabilitation program: a real-life study. Pulmonology 2019;25:71-8.

13. Linn BS, Linn MW, Gurel L. Cumulative illness rating scale. J Am Geriatr Soc 1968;16:622-6.

14. Shah S, Vanclay F, Cooper B. Improving the sensitivity of the Barthel index for stroke rehabilitation. J Clin Epidemiol 1989;42:703-9.

15. Bernabeu-Mora R, Medina-Mirapeix F, Llamazares-Herran E, et al. The short physical performance battery is a discriminative tool for identifying patients with COPD at risk of disability. Int J Chron Obstruct Pulmon Dis 2015;10:2619-26.

16. Guralnik JM, Simonsick EM, Ferrucci L, et al. A short physical performance battery assessing lower extremity function: association with self-reported disability and prediction of mortality and nursing home admission. J Gerontol 1994;49:M85-94.

17. Holland AE, Spruit MA, Troosters T, et al. An official European Respiratory Society/American Thoracic Society technical standard: field walking tests in chronic respiratory disease. Eur Respir J 2014;44:1428-46.

18. Vitacca M, Paneroni M, Baiardi P, et al. Development of a Barthel Index based on dyspnea for patients with respiratory diseases. Int J Chron Obstruct Pulmon Dis 2016;11:1199-206.

19. Bestall JC, Paul EA, Garrod R, et al. Usefulness of the Medical Research Council (MRC) dyspnoea scale as a measure of disability in patients with chronic obstructive pulmonary disease. Thorax 1999;54 581-6.

20. Vitacca M, Carone M, Clini EM, et al. Joint statement on the role of respiratory rehabilitation in the COVID-19 crisis: the Italian position paper. Respiration 2020;99:493-9.

21. Borg G. Psychophysical basis of perceived exertion. Med Sci Sports Exerc 1982;14:377-81

22. Belli S, Balbi B, Prince I, et al. Low physical functioning and impaired performance of activities of daily life in COVID-19 patients who survived hospitalisation. Eur Respir J 2020;56:2002096.

23. Curci C, Pisano F, Bonacci E, et al. Early rehabilitation in postacute COVID-19 patients: data from an Italian COVID-19 Rehabilitation Unit and proposal of a treatment protocol. Eur J Phys Rehabil Med 2020;56:633-41.

24. Curci C, Negrini F, Ferrillo M, et al. Functional outcome after inpatient rehabilitation in post-intensive care unit COVID-19 patients: findings and clinical implications from a real-practice retrospective study. Eur J Phys Rehabil Med 2021;57:443-50.

25. Zampogna E, Migliori GB, Centis R, et al. Functional impairment during post-acute COVID-19 phase: Preliminary finding in 56 patients. Pulmonology 2021;27:452-5.

26. Perera S, Mody SH, Woodman RC, Studenski SA. Meaningful change and responsiveness in common physical performance measures in older adults. J Am Geriatr Soc 2006;54:743-9.

27. Vitacca M, Paneroni M, Peroni R, et al. Effects of a multidisciplinary care program on disability, autonomy, and nursing needs in subjects recovering from acute respiratory failure in a chronic ventilator facility. Respir Care 2014;59:1863-71. 